\title{
Should adjuvant therapy be used in patients with colorectal cancer in pathological stage II?
}

\author{
Rafał Stec
}

In meta-analyses and reliable randomised clinical trials, the most favourable therapeutic gain of adjuvant therapy of patients with colorectal cancer in stage II was estimated at 3\% (in the majority of meta-analyses no statistical significance was). Considering the side effects, especially the mortality rate in chemotherapy (FOLFOX reached $0.5 \%$, and in the case of 5 -fluorouracil, the occurrence of toxicity grade 3 and 4, increased the risk of patient death by approximately $1 \%$ ) seems to be questionable. Because of the lack confirmation of the effect of systemic treatment, an increase of the percentage of cured patients in the second stage has led to the development of several genetic tests determining the genetic signature of colorectal cancer. The aim of these tests is to identify the groups of patients that could potentially achieve the greatest benefit from adjuvant therapy. The most important tests are: "Oncotype DX", "ColoPrint" and "GeneFx". However, at the moment, there is no clear scientific evidence in favour of the standard use of adjuvant chemotherapy in patients with colon cancer in stage II.

NOWOTWORY J Oncol 2017; 67, 6: 375-379

Key words: colorectal cancer, pathological stage II, adjuvant chemotherapy

Currently there is no evidence-based data providing arguments for the application of adjuvant therapy in patients with colorectal cancer in pathological stage II. Below there are arguments against the use of adjuvant chemotherapy in the above clinical situation.

The meta-analysis which points to the lack of benefits resulting from such a treatment pattern is, among others, the "Intergroup" meta-analysis, comprising 7 clinical studies (total number of colorectal cancer patients: 3302), in which the adjuvant therapy regimen was based on treatment with 5-fluorouracil in combination with levamisole or folic acid. The rate of patients with a 5-year DFS (disease-free survival) period in the "N0" patient group receiving adjuvant therapy in comparison with the group of patients without chemotherapy was $76 \%$ vs $72 \%$ respectively $(p=0.0490)$, whilst there was no improvement with regards to OS (overall survival): $81 \%$ vs $80 \%$ respectively $(p=0.1127)$ [1].

In the systematic review (altogether 37 clinical studies and 11 meta-analyses) published by the "Ontario group", the final analysis concerned 4187 patients with colorectal cancer in pathological stage II, who, in the chemotherapy arm, received the regimen based on at least 5-fluorouracil in monotherapy. In the group of patients treated with chemotherapy, DFS improved by $5-10 \%$, which did not translate to a significant improvement of OS $(\mathrm{HR}=0.87$, $95 \% \mathrm{Cl}, 0.75-1.10 ; \mathrm{p}=0.07)[2,3]$.

Likewise, no statistically significant benefit was observed in the "Impact B2" meta-analysis (5 clinical trials, 1016 patients:T3N0) with regards to the improvement of the DFS in patients treated with chemotherapy (regimen 5-fluorouracil and folinic acid) in comparison with patients undergoing observation alone (76\% vs $73 \%$ respectively) and a 5 -year OS (82\% vs $80 \%$ respectively) $[3,4]$.

A slight benefit in the use of adjuvant chemotherapy was observed only in the "QUASAR" clinical study ("QUick And Simple And Reliable"). The study comprised 3238 subjects with colorectal cancer with so-called "uncertain indications for adjuvant therapy" (only 2146 subjects had pathological stage II of colorectal cancer): $71 \%$ had a diagnosis of colon cancer and $29 \%$ of rectal cancer. The chemotherapy consisted of a three or two-drug regimen: 5-fluorouracil with folinic acid with or without the addition of levamisole. The 
improvement in the 5-year OS was obtained in the group with chemotherapy in comparison with the patients under observation alone $(83.9 \%$ vs $81.5 \% ; \mathrm{HR}=0.86,95 \% \mathrm{Cl}$, 0.54-1.19). [3, 5].

The presented analyses included in total more than 10 600 patients with pathological stage II colorectal cancer obtaining uncertain benefit only in one clinical study (the patients enrolled had colorectal cancer and various chemotherapy schemes were applied) at the level of about 3\%.

These conclusions may also be confirmed by the results of a retrospective analysis which included the "SEER-Medicare" ("Surveillance, Epidemiology, and End Results") database from 1992-2005 comprising as many as 24847 patients with pathological stage II colorectal cancer. $75 \%$ of patients had at least one or more negative prognostic factors (hospital admission on an emergency basis on account of colorectal cancer, intestinal perforation, ileus, tumour size T3 or T4, poor differentiation of the tumour, the number of lymph nodes in the specimen $<12$ ), and, in this group, $20 \%$ of subjects received adjuvant chemotherapy. No statistically significant differences were found between the two groups of patients: without negative prognostic factors and untreated with chemotherapy (the 5-year OS was $70.0 \%$ vs $69.5 \%$ respectively; [HR $=1.02 ; 95 \% \mathrm{Cl} ; 0.84-1.25]$ ) and also with at least one or more negative prognostic factors, treated and untreated with chemotherapy (5-year OS was $56.7 \%$ vs $56.1 \%$ respectively; $[\mathrm{HR}=1.03 ; 95 \% \mathrm{Cl}, 0.94-1.13$ ). A significant benefit in the adjuvant treatment was obtained solely by patients with pathological stage III ( $\mathrm{HR}=0.64 ; 95 \%$ $\mathrm{Cl}, 0.60-0.67)$ [6].

The largest published database of patients with pathological stage II colorectal cancer ("National Cancer Data Base"), which finally included the cohort of 153,110 patients, evaluated the influence of adjuvant chemotherapy on the 5-year OS, among others in the group of "high" and "low" risk patients. A significant improvement in OS was related to the use of adjuvant chemotherapy in all patient subgroups irrespective of the presence of histopathological risk factors (poorly or non-differentiated tumour, pT4 tumour, positive surgical margin, $<12$ lymph nodes in the post-operative histopathological specimen), patient age, chemotherapy regimen or even after the correction of the results with regards to independent variables $(H R=0.76$, $p<0.001)$. Although the authors proved the benefits in post-operative chemotherapy, the presented analysis has some significant limitations, which clearly undermine the obtained results. The reduction of the value of the study is related to its retrospective and unplanned character, lack of random selection of the patients and the resulting lack of balance between specific patient groups (large, significant disproportion between specific patient groups: the groups of patients without chemotherapy was 4 times larger), non-stringent procedures concerning the collection of cli- nical data concerning the subjects, doubts concerning the improvement of OS after adjuvant chemotherapy in the "low-risk" patient subgroup, diverse follow-up periods (the enrolment scope was 1998-2011 without the presentation of the data concerning the median period and the scopes of the follow-up period) [7].

Some more published data concerning the efficacy of adjuvant chemotherapy applied to patients with pathological stage II colorectal cancer are taken from a Dutch register (The Netherlands Cancer Registry) covering the period from 2008-2012. The analysis comprised 10,935 patients, including 4940 (45\%) with the presence of risk factors (poorly or non-differentiated tumour, pT4 tumour, emergency surgery, and or $<10$ lymph nodes in the post-operative histopathological specimen) out of which adjuvant therapy was received by 790 patients (16\%). A significant improvement in the 3-year OS was obtained only in the groups of patients with pT4 cancer, who received chemotherapy in comparison with the group of patients under observation only (91\% vs $73 \%$, $\mathrm{HR}=0.43,95 \% \mathrm{Cl} 0.28-0.66$ ) [8]. The results of the Dutch registry do not differ significantly from those presented in the "National Cancer Data Base" or in "SEER-Medicare", which definitely undermines the credibility of such analyses.

Also no efficacy improvement was obtained in the case of chemotherapy applied in pathological stage II of colorectal cancer consisting of the addition of oxaliplatin to the two-drug regimen (5-fluorouracil and folinic acid). In the "MOSAIC" clinical study in which about $40 \%$ of subjects had pathological stage II colorectal cancer (899 patients), the 10-year OS was, in the case of the group treated with chemotherapy with oxaliplatin added (FOLFOX 4) $78.4 \%$ vs $79.5 \%$ for the group treated solely with 5 -fluorouracil and folinic acid, yet without obtaining statistical significance $(H R=1.004,95 \% \mathrm{Cl} 0.744-1.354 ; \mathrm{p}=0.981)$. Similarly, no significant improvement of the OS was observed either in the "high risk" group with pathological stage II colorectal cancer ( $\mathrm{HR}=0.895 ; 95 \% \mathrm{Cl}, 0.606-1.323 ; \mathrm{p}=0.578)$, or in the "low risk" subgroup $(\mathrm{HR}=1.168 ; 95 \% \mathrm{Cl}, 0.730-1.870$, $\mathrm{p}=0.515$ ) [9].

It was not clearly determined whether the patients from the "high risk" group should receive chemotherapy in a situation when the post-operative histopathological assessment reveals one, two or a few adverse prognostic factors, which proves the lack of consistency among the recommendations of international committees and societies with regards to the use of chemotherapy in pathological stage Il of colorectal cancer, and undoubtedly illustrates how controversial this treatment is [3]. The most significant factors influencing treatment decisions are presented in Table I.

With regards to the lack of any reliable confirmation on the influence of post-operative systemic treatment on the increase in the rate of cured patients with pathological stage Il of colorectal cancer, a number of genetic tests have been 
Table I. Indications for adjuvant chemotherapy in patients with pathological stage II of colorectal cancer according to various scientific committees and societies

\begin{tabular}{|c|c|c|c|c|c|c|c|}
\hline The name of a scientific committee or a society & ASCO & NCCN & ASCRS & $\mathrm{NCl}$ & ESMO & JSCCR & NHMRC \\
\hline $\begin{array}{l}\text { General remark: "for patients with a high risk of disease } \\
\text { recurrence". }\end{array}$ & & & & $\mathrm{X}$ & & $x$ & \\
\hline $\begin{array}{l}\text { Too small number of lymph nodes in the histopathological } \\
\text { specimen }\end{array}$ & $X$ & $X$ & & $X$ & $X$ & & \\
\hline$<12$ lymph nodes in the histopathological specimen & & $X$ & & & $\mathrm{X}$ & & \\
\hline Tumour pT4 & $x$ & & $x$ & $X$ & $\mathrm{X}$ & & $X$ \\
\hline Tumour perforation & $\mathrm{X}$ & $X$ & $\mathrm{X}$ & & $\mathrm{X}$ & & $X$ \\
\hline lleus & & $\mathrm{X}$ & & & $x$ & & $x$ \\
\hline Poorly or non-differentiated tumour: G3, G4 & $X$ & $X$ & $x$ & $X$ & $X$ & & $X$ \\
\hline Lymphatic vessels involvement & & $x$ & $x$ & & $x$ & & \\
\hline Blood vessels involvement & & $X$ & $X$ & & $X$ & & \\
\hline Perineural invasion & & $X$ & $X$ & & $X$ & & \\
\hline Positive surgical margin & & $\mathrm{X}$ & & & & & \\
\hline Comorbidities & & $X$ & & & & & \\
\hline Estimation of predicted survival & & $X$ & & & & & \\
\hline Molecular tests concerning DNA disorders of the cancer & & $X$ & & & & & \\
\hline The possibility of participation in a clinical study & & & $X$ & & & & \\
\hline Obtaining informed consent for the treatment & & & & & & $\mathrm{X}$ & \\
\hline
\end{tabular}

ASCO (American Society of Clinical Oncology [in collaboration with the Cancer Care Ontario Practice Guideline Initiative]), ASCRS (American Society of Colon and Rectal Surgeons), ESMO (European Society for Medical Oncology), JSCCR (Japanese Society for Cancer of the Colon and Rectum), NCCN (National Comprehensive Cancer Network), NCI (National Cancer Institute), NHMRC (Cancer Council Australia/Australian Cancer Network. National Health and Medical Research Council)

worked out which specify the gene signature of colorectal cancer. The purpose of these tests is the identification of the group of patients who could potentially benefit most from such treatment. The most important tests are: "Oncotype DX" (12-genes), "ColoPrint" (18-gene) and "GeneFx" (482-genes) [3].

Currently the test "Oncotype DX", which was worked out and validated on the basis of a few studies, such as "OUASAR", "CALGB 9581" ("Cancer and Leukemia Group B") and "NSABP C-07" ("National Surgical Adjuvant Breast and Bowel Project C-07") is the most widely used. On the basis of the analysis of the dependence of the correlation of genes and the recurrence score (RS), some algorithms have been specified with a purpose to assess the risk of recurrence of colorectal cancer and the benefits from the use of adjuvant chemotherapy. On the basis of these algorithms (based on the analysis of the patients from the "QUASAR" study treated only with surgery), the patients belonging to specific groups may be identified: low risk of recurrence (3-year recurrence risk is $12 \%$ ), moderate risk (3-year recurrence risk is $18 \%$ ), high risk (3-year recurrence risk is 22\%). In an independent clinical "validation" study (patients from the "NSABP C-07" study) the mean 5-year risk of disease recurrence, after the application of adjuvant chemotherapy containing 5-fluorouracil, was, respectively: $9 \%$ in low risk groups $(95 \% \mathrm{Cl}$, $6-13 \%), 13 \%$ in medium risk groups $(95 \% \mathrm{Cl}, 8-17 \%)$ and $18 \%$ in high risk groups $(95 \% \mathrm{Cl}, 12-25 \%)$. The benefits in the application of adjuvant therapy with regards to the 3-year risk of recurrence, as specified on the basis of the "OUASAR" study, after a 5-year treatment period were 3\%, $5 \%$ and $4 \%$ respectively. An important drawback of this test is the price: 3280 USD, which makes its wide use in clinical practice impossible, as well as the lack of prospective evaluation of the influence of chemotherapy on the reduction of the risk of recurrence and the improvement of overall survival [3, 10-12].

Another test applied for the identification of patients in specific risk groups is "ColoPrint". On the basis of a non-randomised, cohort "validation" study (114 samples), two risk groups were specified: a low risk group, in which a 5-year relapse-free survival (RFS) and 5-year metastasis free survival period (MFS) concerned $87.6 \%$ and $94.9 \%$ of patients respectively and the high risk group in which the 5 -year RFS and 5-year MFS concerned $67.2 \%$ and $80.6 \%$ of patients respectively. In spite of promising results, the "ColoPrint" test has also a number of significant drawbacks. Apart from the high cost of its application, some doubt is raised by the small number of patients included in the "validation" study with varied pathological stages - from I to III, the necessity of obtaining fresh-frozen tumour tissues for molecular analyses, the lack of prospective evaluation of the influence of chemotherapy on the reduction of RFS and MFS risks or on the improvement of overall survival in specific study groups and the possibility of distortion of the results by means of 
including a significant number of tumours with the BRAF or MSI gene mutation into the study $[3,13,14]$.

Another test which may potentially be used for the identification of recurrence risk in patients with pathological stage II of colorectal cancer is a "GeneFx", a multi-gene test, on the basis of which (the analysis of the paraffin-embedded samples and clinical data obtained from 144 patients from 12 centres) the 5 -year recurrence risk and 5 -year OS were evaluated in the validation study. This allowed for distinguishing between groups with a low and high risk of disease recurrence. In the low-risk group, the 5-year recurrence risk was $30 \%$, and the rate of 5 -year survival was $-75 \%$ of patients, whilst in the high risk group it was 55\% and 60\% respectively. The patients in the high risk group of disease recurrence in comparison with patients in the low risk group, had a two and a half-fold higher risk of disease recurrence $(H R=2,53 ; p<0,001)$. The results were again evaluated on the basis of histopathological material concerning 393 patients who were treated within the "CALGB 9581" clinical study. 177 patients (45\%) were categorised as patients with a low risk of recurrence and $216(55 \%)$ as patients with a high risk of recurrence. The risk of disease recurrence in the group of patients classified as a group with the high risk of recurrence, (in comparison with the patients from the group with a low risk of recurrence) was statistically significant, as it was twice as high $(\mathrm{HR}=2.13 ; 95 \% \mathrm{Cl}, 1.3-3.5)$, whilst the 5 -year disease-free interval was $82 \%$ versus $91 \%$ in the specific groups respectively.
A disadvantage of this test, similarly to the case of the previously described ones, is the high cost and the lack of a prospective evaluation of the influence of chemotherapy on the reduction of the risk of disease recurrence and the improvement of overall survival $[3,15,16]$. The genetic tests are summarised in Table II.

Another important aspect which determines the treatment possibilities, apart from efficiency, is toxicity. In the case of adjuvant chemotherapy, in patients with colorectal cancer, the pathological stage of the disease is connected with a significant increase (from $15 \%$ to even $53 \%$ ) in the risk of adverse reactions in the GI tract (nausea, vomiting, alimentary tract mucositis, diarrhoea), a risk of leukopenia (from $8 \%$ to $30 \%$ ) or a neuropathy risk, which may concern even as many as $84 \%$ of patients treated with oxaliplatin, which definitely leads to a deterioration in the quality of life. Nevertheless, the most important complication of chemotherapy is the patient's death. In the group of patients treated with the FOLFOX regimen, the mortality rate was $0.5 \%$, whereas in the case of 5 -fluorouracil, third and fourth degree toxicity increased the risk of a patient's death by about $1 \%$. Moreover, adjuvant treatment requires frequent patient visits to hospital, in chemotherapy wards (either on an outpatient or inpatient basis) for a period from 6 to 8 months, which directly affects the family, social and professional life of patients [3, 17-20]. The costs of chemotherapy must not be forgotten either, for example in the "QUASAR" study, it was estimated that this costs ranged from 2000 GBP

Table II. The available genetic tests used for the evaluation of the prognoses of colorectal cancer patients

\begin{tabular}{|c|c|c|c|}
\hline Test name & Oncotype Dx & ColoPrint & GeneFx \\
\hline $\begin{array}{l}\text { The number of examined } \\
\text { genes }\end{array}$ & 12 & 18 & 482 \\
\hline "validation" studies & "QUASAR"“NSABP C-07" & $\begin{array}{l}\text { A study on an independent } \\
\text { patient cohort. Prospective } \\
\text { analysis of risk stratification } \\
\text { (PARSC) }\end{array}$ & $\begin{array}{l}\text { A study on an independent patient cohort, } \\
\text { "CALGB 9581" }\end{array}$ \\
\hline Results & $\begin{array}{l}\text { 3-year recurrence risk "QUASAR": } \\
\text { - } \text { the group of low risk of } \\
\text { recurrence: } 12 \% \\
\text { - } \text { the group of medium risk of } \\
\text { recurrence: } 18 \% \\
\text { - } \text { the group of high risk of } \\
\text { recurrence: } 22 \% \\
\text { "Validation" study ("NSABP C-07 ") } \\
\text { after the application of adjuvant } \\
\text { chemotherapy containing } \\
\text { 5-fluorouracil, the mean risk of } \\
\text { recurrence in the specific groups } \\
\text { was: } \\
\text { - low risk: } 9 \% \text { ( } 95 \% \mathrm{Cl}, 6-13 \%) \\
\text { - medium risk: } 13 \%(95 \% \mathrm{Cl}, \\
8 \%-17 \%) \\
\text { - } \text { high risk: } 18 \%(95 \% \mathrm{Cl}, 12- \\
\quad 25 \%)\end{array}$ & $\begin{array}{l}\text { 5-year relapse free survival } \\
\text { (RFS): } \\
\text { - low risk: } 87.6 \% \\
\text { - high risk: } 67.2 \% \\
\text { 5-year metastases free } \\
\text { survival (MFS): } \\
\text { - low risk: } 94.9 \% \\
\text { - high risk: } 80.6 \%\end{array}$ & $\begin{array}{l}\text { An independent cohort: } \\
\text { 5-year risk of recurrence: - low risk group: } 30 \% \text {, } \\
\text { - high risk group: } 55 \% \\
\text { The group of high risk of disease recurrence } \\
\text { in comparison with the group of low risk of } \\
\text { recurrence had a two and half-fold higher risk of } \\
\text { disease recurrence, } \mathrm{HR}=2.53 ; \mathrm{p}<0,001) \\
5 \text {-year OS: } \\
\text { - low risk group: } 75 \% \\
\text { - high risk group: } 60 \% \\
\text { "CALGB } 9581 \text { " } 393 \text { patients: } 177 \text { patients ( } 45 \%) \\
\text { a low disease recurrence risk group, } 216 \text { patients } \\
\text { (55\%) a high disease recurrence risk group; the } \\
\text { high disease recurrence risk group in comparison } \\
\text { with the low risk group had a twofold higher risk } \\
\text { of disease recurrence (HR = } 2.13 ; 95 \% \mathrm{Cl}, 1.3-3.5 \text { ); } \\
\text { the } 5 \text {-year recurrence-free interval was, in specific } \\
\text { groups: } 82 \% \text { vs } 91 \% \text { relatively }\end{array}$ \\
\hline
\end{tabular}


to $3000 \mathrm{GBP}$ per patient $[3,5]$. The social costs or the costs of the lack of professional activity of the patients during systemic post-operative therapy were not assessed.

To sum up, currently there is no reliable evidence which might definitely argue for the use of adjuvant chemotherapy in colorectal cancer patients with pathological stage II of the disease. The majority of scientific data argue against such treatment patterns, as it causes only adverse effects without any significant improvement in survival indicators.

\section{Reply}

Some adverse prognostic factors qualifying patients to the "high risk" group are not related to tumour biology, but only with suboptimal surgery. These factors comprise: the number of resected lymph nodes below 12 , as well as positive or not adequate surgical margins (e.g. R1 resection). Therefor it seems more important to maintain standards in surgical treatment than to use adjuvant chemotherapy. Some reliable studies with randomisation and meta-analyses show that the most optimistic therapeutic gain (improvement of 5-year survival) from adjuvant treatment in patients with pathological stage II colorectal cancer was established to be $3 \%$ (in the majority of meta-analyses no statistical significance was gained). Yet, given the adverse effects, and, in particular, the mortality rate with the chemotherapy used (with the FOLFOX regimen it was $0.5 \%$, and in the case of 5-fluorouracil with the third and fourth toxicity grade it increased the mortality risk by about $1 \%$ ), such treatment patterns seems to be highly doubtful.

\section{Conflict of interest: none declared}

\section{Rafał Stec, MD, PhD}

Military Institute of Medicine

Department of Oncology

ul. Szaserów 128

04-141 Warszawa, Poland

e-mail:drrafals@wp.pl

Received \& Accepted: 10 Apr 2017

Based on the presentation at the $\mathrm{V}$ Annual Conference of the Nowotwory Journal of Oncology, 'Oncological Debates', held in Warszawa, 7-8th April 2017
References (the most important items)

1. Gill S, Loprinzi CL, Sargent DJ et al. Pooled analysis of fluorouracil-based adjuvant therapy for stage II and III colon cancer: who benefits and by how much? J Clin Oncol 2004; 22: 1797-1806.

2. Figueredo A, Charette ML, Maroun J et al. Adjuvant therapy for stage II colon cancer: a systematic review from the Cancer Care Ontario Program in evidence-based care's gastrointestinal cancer disease site group. $J$ Clin Oncol 2004; 22: 3395-3407.

3. Fang $\mathrm{SH}$, Efron JE, Berho ME et al. Dilemma of stage II colon cancer and decision making for adjuvant chemotherapy. J Am Coll Surg 2014; 219: 1056-1069.

4. [No author listed]. Efficacy of adjuvant fluorouracil and folinic acid in colon cancer. Lancet 1995; 345: 939-944.

5. Gray R, Barnwell J, McConkey C et al. Adjuvant chemotherapy versus observation in patients with colorectal cancer: a randomised study. Lancet 2007; 370: 2020-2029.

6. O'Connor ES, Greenblatt DY, LoConte NK et al. Adjuvant chemotherapy for stage II colon cancer with poor prognostic features. J Clin Oncol 2011; 29: 3381-3388.

7. Casadaban L, Rauscher G, Aklilu M et al. Adjuvant chemotherapy is associated with improved survival in patients with stage II colon cancer. Cancer 2016; 122: 3277-3287.

8. Verhoeff SR, van Erning FN, Lemmens VE et al. Adjuvant chemotherapy is not associated with improved survival for all high-risk factors in stage II colon cancer. Int J Cancer 2016; 139: 187-193.

9. André T, de Gramont A, Vernerey D et al. Adjuvant fluorouracil, leucovorin, and oxaliplatin in stage II to III colon cancer: updated 10-year survival and outcomes according to BRAF mutation and mismatch repair status of the MOSAIC study. J Clin Oncol 2015; 33: 4176-4187.

10. Kelley RK, Venook AP. Prognostic and predictive markers in stage II colon cancer: is there a role for gene expression profiling? Clin Colorectal Cancer 2011; 10: 73-80.

11. Yothers $\mathrm{G}, \mathrm{O}^{\prime} \mathrm{C}$ onnell MJ, Lee $\mathrm{M}$ et al. Validation of the 12-gene colon cancer recurrence score in NSABP $\mathrm{C}-07$ as a predictor of recurrence in patients with stage II and III colon cancer treated with fluorouracil and leucovorin (FU/LV) and FU/ LV plus oxaliplatin. J Clin Oncol 2013; 31: 4512-4519.

12. http://www.xconomy.com/san-francisco/2011/09/30/genomic-healthwins-medicare-coverage-for-high-priced-colon-cancer-test/.

13. Salazar R, Roepman $\mathrm{P}$, Capella $\mathrm{G}$ et al. Gene expression signature to improve prognosis prediction of stage II and III colorectal cancer. J Clin Oncol 2011; 29: 17-24.

14. Maak M, Simon I, Nitsche U et al. Independent validation of a prognostic genomic signature (ColoPrint) for patients with stage II colon cancer. Ann Surg 2013; 257: 1053-1058.

15. Kennedy RD, Bylesjo M, Kerr P et al. Development and independent validation of a prognostic assay for stage II colon cancer using formalin-fixed paraffin-embedded tissue. J Clin Oncol 2011; 29: 4620-4626.

16. Niedzwiecki D, Frankel W, Venook AP. Association between ColDx assay result and recurrence-free interval in stage II colon cancer patients on CALGB (Alliance) 9581. J Clin Oncol 2014; 32:(suppl 3; abstr 455).

17. Andre $T$, Boni $C$, Navarro $M$ et al. Improved overall survival with oxaliplatin, fluorouracil, and leucovorin as adjuvant treatment in stage II or III colon cancer in the MOSAIC trial. J Clin Oncol 2009; 27: 3109-3116.

18. Rothenberg ML, Meropol NJ, Poplin EA et al. Mortality associated with irinotecan plus bolus fluorouracil/leucovorin: summary findings of an independent panel. J Clin Oncol 2001; 19: 3801-3807.

19. Deptała A, Omyła-Staszewska J. Leczenie adiuwantowe raka jelita grubego. In: Rakjelita grubego, Deptała A i Wojtukiewicz MZ (ed.). Poznań: Termedia Wydawnictwa Medyczne, 2012.

20. Lewis C, Xun P, He K. Effects of adjuvant chemotherapy on recurrence, survival, and quality of life in stage II colon cancer patients: a 24-month follow-up. Support Care Cancer 2016; 24: 1463-1471. 